\title{
Global and regional trends in COPD mortality, 1990-2010
}

\author{
Peter G.J. Burney ${ }^{1,2,3}$, Jaymini Patel ${ }^{1}$, Roger Newson ${ }^{1}$, Cosetta Minelli ${ }^{1}$ and \\ Mohsen Naghavi ${ }^{4}$
}

Affiliations:

${ }^{1}$ National Heart and Lung Institute, Imperial College, London, UK.

${ }^{2}$ Wellcome Centre for Global Health, Imperial College, London, UK.

${ }^{3}$ MRC-PHE Centre for Environment and Health, Imperial College, London, UK.

${ }^{4}$ Institute for Health Metrics and Evaluation, Seattle, WA, USA.

\section{Correspondence:}

Peter G.J. Burney, Respiratory Epidemiology, Occupational Medicine and Public Health, National Heart and Lung Institute, Imperial College, Emmanuel Kaye Building, 1 Manresa Road, London, SW3 6LR, UK.

E-mail: p.burneydimperial.ac.uk

ABSTRACT Between 1990 and 2010, chronic obstructive pulmonary disease (COPD) moved from the fourth to third most common cause of death worldwide.

Using data from the Global Burden of Disease programme we quantified regional changes in the number of COPD deaths and COPD mortality rates between 1990 and 2010. We estimated the proportion of the change that was attributable to gross national income per capita and an index of cumulative smoking exposure, and quantified the difference in mortality rates attributable to demographic changes.

Despite a substantial decrease in COPD mortality rates, COPD deaths fell only slightly, from three million in 1990 to 2.8 million in 2010, because the mean age of the population increased. The number of COPD deaths in 2010 would have risen to 5.2 million if the age- and sex-specific mortality rates had remained constant. Changes in smoking led to only a small increase in age- and sex-specific mortality rates, which were strongly associated with changes in gross national income.

The increased burden of COPD mortality was mainly driven by changes in age distribution, but ageand sex-specific rates fell as incomes rose. The rapid response to increasing affluence suggests that changes in COPD mortality are not entirely explained by changes in early life.

@ERSpublications

COPD age-standardised mortality rates decrease as incomes increase, but COPD deaths increase as population ages http://ow.ly/I66ND

Support statement: This work was supported by funding from the Bill and Melinda Gates Foundation. J. Patel is supported by the Wellcome Trust (085790/Z/08/Z). Funding information for this article has been deposited with FundRef.

Conflicts of interest: None declared.

Copyright @ERS 2015 


\section{Introduction}

Global patterns of mortality are changing, mainly due to three related transformations: 1) an epidemiological transition driven by change in global risks, with falling death rates in childhood associated with infectious disease; 2) a demographic transition with more people surviving into later life, and 3) a healthcare transition with improved effectiveness of both preventive and curative or palliative treatments. The reassessment of the Global Burden of Disease programme in 2010 identified chronic obstructive pulmonary disease (COPD) as the third most common cause of death worldwide [1], up from fourth in 1990 [2]. Understanding the reasons for this is important in order to identify potential public health interventions. In particular, we need to know whether this change has been caused by an increase in the mortality rate or by an increase in the absolute number of deaths due to increased prevalence, for example associated with ageing in the population. Alternatively, the change may be a relative one, with COPD mortality having declined less than mortality from other causes.

Cigarette smoking is regarded as the most important cause of COPD, with other inhaled hazards from occupational, domestic or outdoor sources thought to play an important role [3]. However, in a study of mortality in local authorities in England and Wales, BARKER and OSMOND [4] noted that there was a strong association between childhood deaths from pneumonia and bronchitis in the 1920s and deaths from "COPD" in the 1970s, with high death rates from both conditions being strongly associated with poverty. As this association was not noted for lung cancer, this relationship was unlikely to be strongly confounded by cigarette smoking. Subsequently, by following up a cohort of men born in the 1920s, with very complete health records from the first year of life, the authors showed associations between early life factors and death from COPD and adult lung function, again associations that were not explained by smoking habits [5]. More recently, the Burden of Obstructive Lung Disease (BOLD) study has shown a strong ecological association between national levels of poverty and the prevalence of both a low forced vital capacity (FVC) and national COPD mortality rates [6].

Many descriptions of national and regional mortality trends over time have been published previously [7-14]. These have reported variable trends, mostly at a local level, and have rarely analysed the drivers of change. In this study we have examined trends in COPD mortality rates for all 21 Global Burden of Disease regions. We have assessed the impact of population changes on the number of COPD deaths, the associations between changes in age- and sex-specific mortality rates from COPD, and changes in both national income and smoking. By analysing trends within regions over time, we have avoided much of the confounding between national income and other factors that vary across regions, which is inevitable in purely cross-sectional analyses.

\section{Methods}

Between 1990 and 1999, mortality data were collected by country and year in countries allocated to 21 Global Burden of Disease regions from 566 vital registration sources, 27 surveillance reports and one verbal autopsy report. Between 2000 and 2010, mortality data were collected by country and year in the same regions from 894 vital registration sources, 24 surveillance reports and eight verbal autopsy reports. Our definition of COPD is based on in International Classification of Diseases- 9 codes 490-492, 494 and 496, and International Classification of Diseases-10 codes J40-J44, J47. We used previously developed algorithms [15] to improve the quality of the information on mortality by redistributing vague and unsatisfactory causes of death (garbage codes) to more likely causes of death using similar methods in all countries and years. The selection of the model used to assign deaths to specific causes was based on its out-of-sample predictive validity [15]. Our model of COPD mortality assigns each death to a single cause.

We analysed COPD mortality rates for 1990 and 2010 for each region and each age- and sex-specific group, limiting the analyses to people aged $\geqslant 40$ years (18 groups per region, nine groups for age and two groups for sex). We used information on regional mortality rates from lung cancer for the same age and sex groups to create a proxy for smoking exposure, based on a modification of the method proposed by Pето et al. [16]. This uses the strong association between smoking and lung cancer mortality to estimate the cumulative lifetime exposure to tobacco. Briefly, a common baseline mortality rate from lung cancer was assumed in the absence of smoking and estimated from the age- and sex-specific lung cancer mortality rates among nonsmokers in the American Cancer Society's Cancer Prevention Study II. Subtracting these figures from the age- and sex-specific lung cancer mortality rates recorded in the Global Burden of Disease data, we derived an index of cumulative smoking exposure for each group. This index, referred to as the smoking index, is expressed as a rate per 100000 . Gross national income (GNI) per capita was estimated in US dollars and adjusted for purchasing power parity using data from the World Bank [17]. The average GNI for each country in the region was weighted by the total population of each country to provide a regional value for 1990 and 2010. To compare COPD mortality rates across regions, we estimated age-standardised mortality rates by applying the regional age-specific rates to a standard 
world population covering the same age span $(\geqslant 40$ years). For each region, the number of deaths that would have been expected in 2010 if the population had been the same as in 1990 was calculated, by taking the age- and sex-specific COPD mortality rates for 2010 and applying them to the age and sex population distribution of 1990. The difference between this figure and the actual number of deaths in 2010 gave the change in deaths due to changes in the age and sex structure and size of the population.

To analyse the effects of GNI and smoking on COPD mortality rates in 1990 and 2010, and their impact on the change in COPD mortality between the two time-points, we used multilevel regression analysis accounting for the clustering of the data within regions. We first analysed the COPD mortality rate against GNI and smoking index separately for 1990 and 2010, adjusting for age and sex, with log transformation of the mortality rate, GNI and smoking index. In order to log transform the smoking index we added a small constant (180 per 100000) to ensure that all values were positive. We then analysed the change in COPD mortality rates using a multilevel linear regression model with the log of the 2010:1990 mortality rate ratio as the outcome and the corresponding 2010:1990 log ratios for GNI and smoking index as linear predictors, and adjusting for age and sex as categorical predictors with additive effects. As a sensitivity analysis, we reran the analysis after dropping the regions where the change in GNI had been highest, namely East Asia, South-East Asia, Andean Latin America, and southern Latin America.

We estimated the population attributable fraction of the change in COPD mortality for GNI and smoking, i.e. the percentage of change in COPD mortality between 1990 and 2010 that was attributable to changes in GNI and smoking, using the programme margins in Stata (StataCorp., College Station, TX, USA) [18]; all analyses were undertaken in Stata 12.2.

\section{Results}

Most deaths from COPD occur in low-income regions including East, South and South-East Asia and central Africa, where age-standardised rates are also higher (fig. 1). Globally, the total number of COPD deaths fell from three million in 1990 to 2.8 million in 2010, a fall of 5.5\%, whereas the expected number of age- and sex-specific rates were maintained at the 1990 levels, which was very much higher at 5.2 million (table 1). There was a clear fall in age- and sex-specific rates for all groups between 1990 and 2010 (fig. 2a), and COPD mortality fell in all age groups, with the greatest relative declines in younger people (fig. 2b). There was substantial variation in the 2010:1990 mortality rate ratio but clear reductions in all regions, except the North America high-income region where the mortality rate remained stable (fig. 3 ).

Changes in smoking index varied widely between regions, from a reduction of $15.3 \%$ in South Sub-Saharan Africa to an increase of $14.5 \%$ in East Asia (table 2). GNI increased in all regions over this

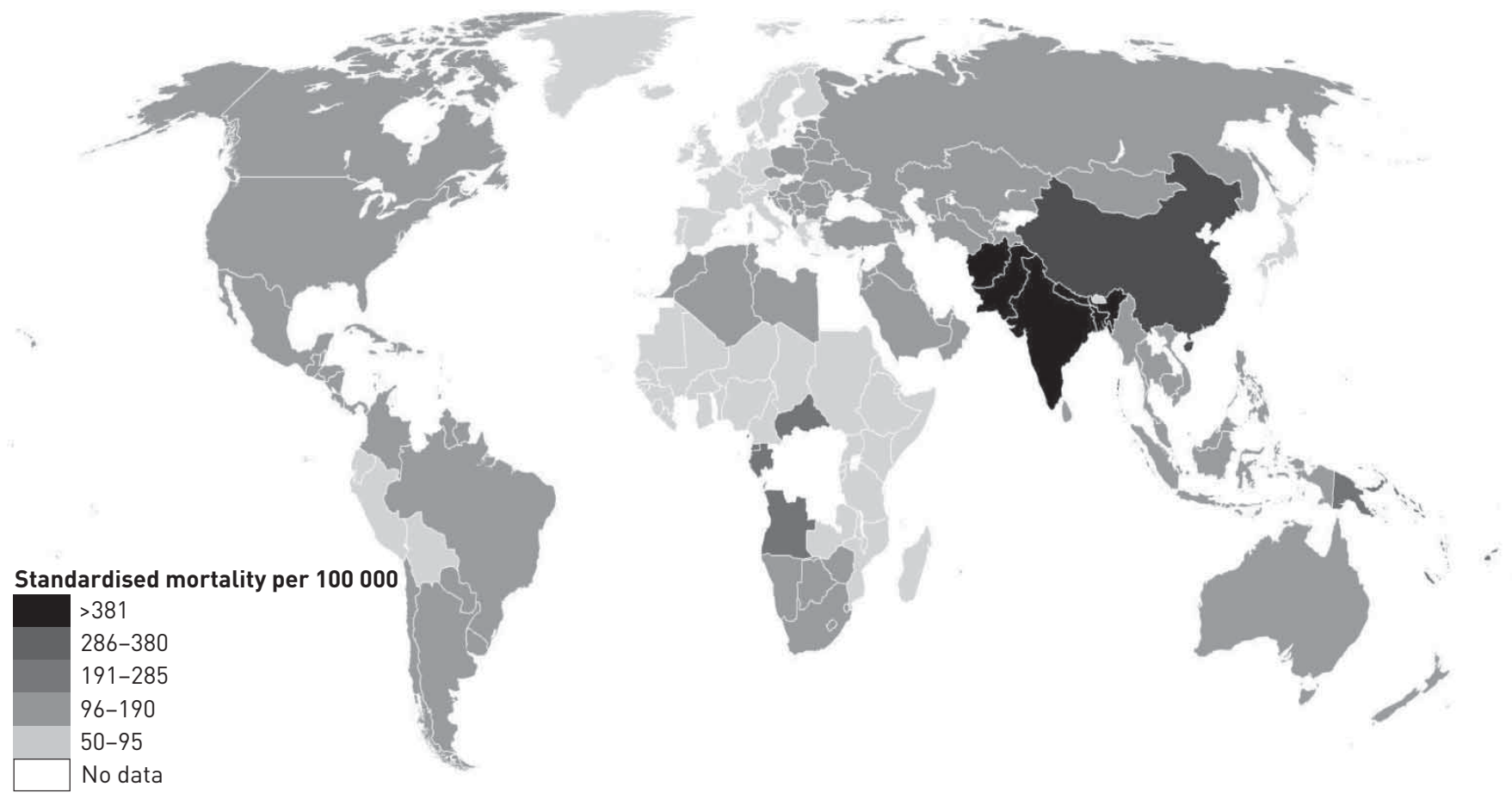

FIGURE 1 Age- and sex-adjusted chronic obstructive pulmonary disease mortality in 2010. 
TABLE 1 Chronic obstructive pulmonary disease (COPD) deaths, total population and age-standardised COPD mortality rates by region and year

Region
1990

\begin{tabular}{|c|c|c|}
\hline $\begin{array}{l}\text { COPD } \\
\text { deaths }\end{array}$ & Population & $\begin{array}{c}\text { Age-standardised } \\
\text { COPD mortality per } \\
100000\end{array}$ \\
\hline
\end{tabular}

2010

\begin{tabular}{cc}
\hline COPD deaths & Population \\
& $\begin{array}{c}\text { Age-standardised } \\
\text { COPD mortality per } \\
100000\end{array}$
\end{tabular}

Expected COPD deaths in $2010^{\#}$

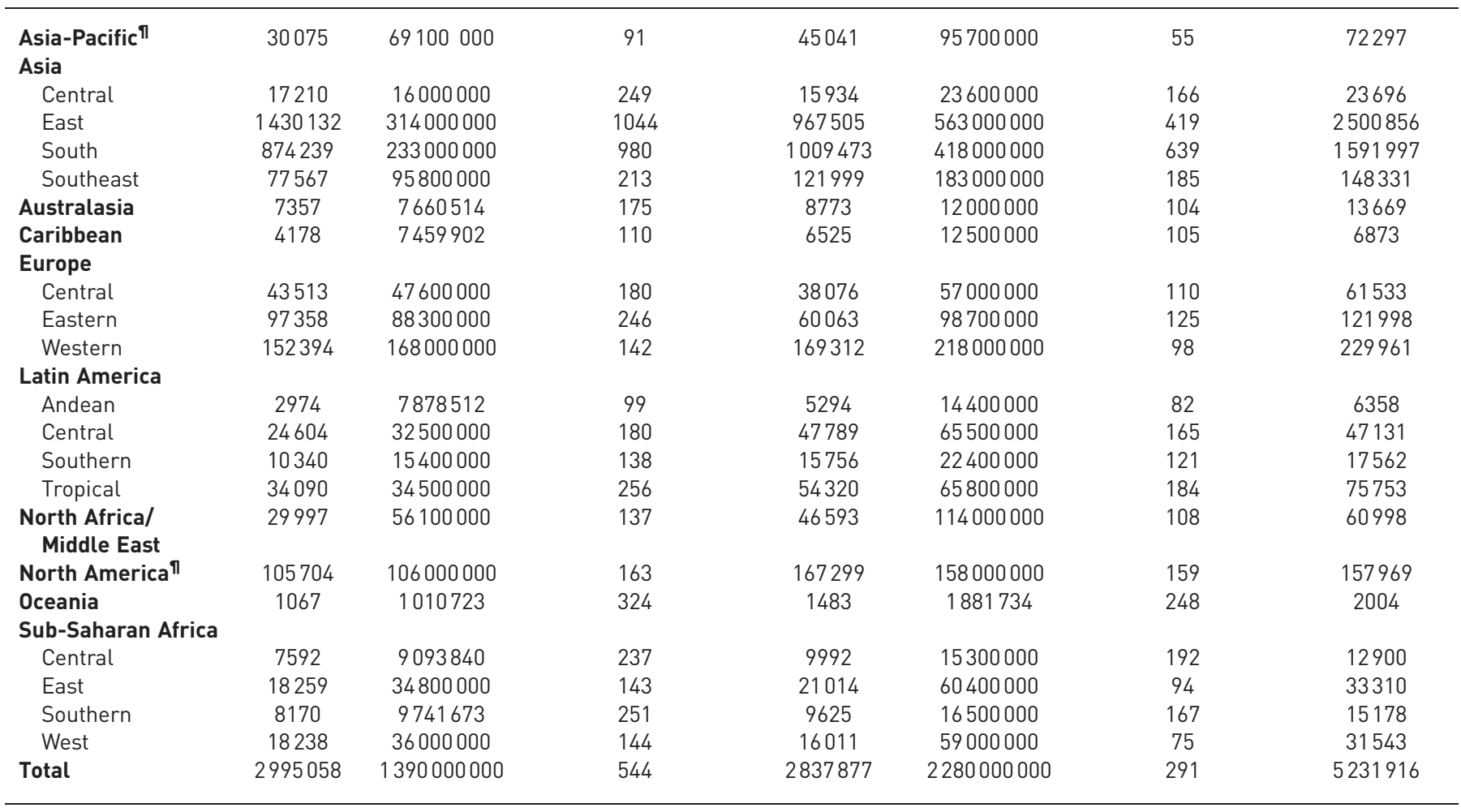

Data are presented as $n{ }^{\#}$ : calculated as the number of deaths expected in 2010 if the population had been the same as in 1990 in terms of age and sex; "? high income.

period of time, except in West Sub-Saharan Africa. In most regions this increase was substantial and in East Asia it was $>700 \%$. Plotting age-standardised COPD mortality rates by GNI showed a negative association. Because this appeared nonlinear we transformed GNI to a log scale.

Multilevel regression analysis, adjusting for age and sex, showed a highly statistically significant association of smoking index and GNI with COPD mortality rate for both years (table 3). Since the model is a log-log
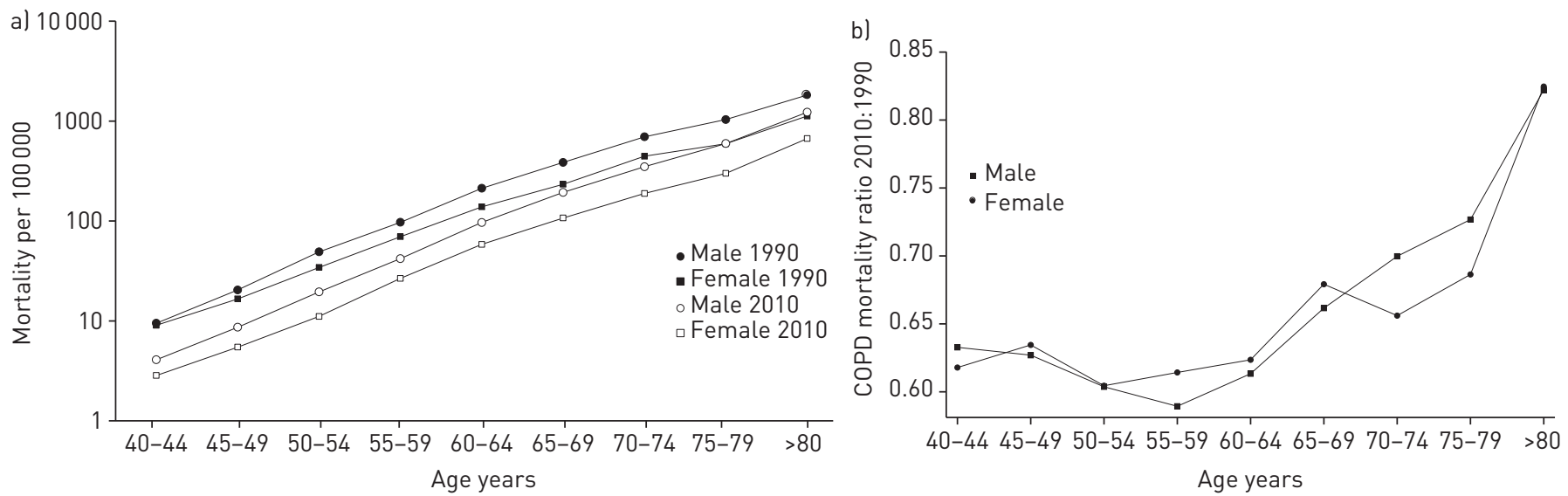

FIGURE 2 a) Chronic obstructive pulmonary disease (COPD) mortality rates for 1990 and 2010, and b) COPD mortality ratios for 2010:1990 according to sex and age. 


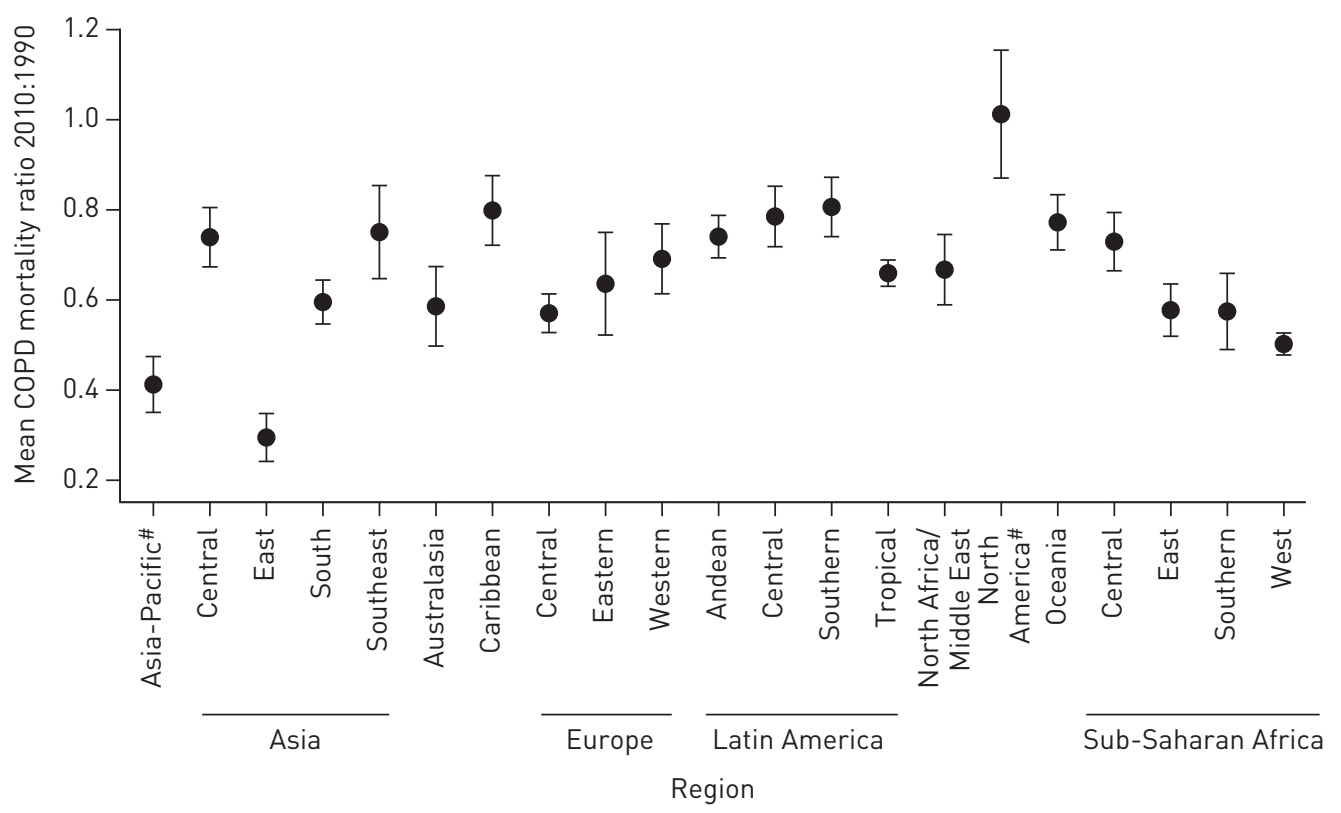

FIGURE 3 Mean age- and sex-specific chronic obstructive pulmonary disease (COPD) mortality ratios for 2010:1990 for each of the 21 regions. ${ }^{*}$ : high income.

regression (exposure and outcome both log transformed), the coefficients for GNI and smoking represent the percentage change in COPD mortality rate given a certain percentage change in GNI or smoking, while keeping all other variables constant. For example, in the 1990 analysis, if we consider the effect of a $50 \%$ increase in exposure ( 1.5 times), the GNI coefficient of -0.47 means a decrease in mortality rate of $17 \%\left(1.5^{-0} .{ }^{47}=0.83\right.$, i.e. $17 \%$ reduction $)$; similarly, the smoking coefficient of 0.43 means a $19 \%$ increase in mortality rate. Corresponding figures for 2010 were a $12 \%$ (95\% CI 3-19\%) decrease for a 50\% increase in GNI and a $17 \%$ (95\% CI 12-21\%) increase for a 50\% increase in smoking index. As expected, for both 1990 and 2010 the model showed COPD mortality rates were higher in older age groups and in men, independent of smoking.

The multilevel regression analysis of the ratio of COPD mortality rates between 2010 and 1990, also analysed as a log-log model, showed a $13 \%$ decrease in mortality rate for a $50 \%$ increase in GNI from 1990 to 2010 ( $\mathrm{p}=0.02)$. The effect of change in smoking index (2010:1990 mortality rate ratio) on change in COPD mortality was also statistically significant, although its magnitude was much smaller; a $0.16 \%$ (95\% CI $0.08-0.17 \%$ ) increase in COPD mortality rate for a $50 \%$ increase in smoking index (table 3 ). In agreement with figure 2, the multilevel regression model showed large between-region variation in COPD mortality, with an intraclass correlation coefficient of 0.69 . When we dropped the four regions with the highest change in GNI from the analysis we obtained the same coefficient for the smoking index and only a small change in the coefficient for GNI, with a $16 \%(95 \%$ CI $4-25 \%)$ decrease in COPD mortality rate for a 50\% increase in GNI from 1990 to 2010. This remained statistically significant.

Finally, we estimated the percentage change in COPD mortality that was attributable to the change in GNI and smoking exposure. Changes in GNI and smoking exposure accounted for a $26.2 \%$ (95\% CI $4.7-47.7 \%$ ) and for $3.9 \%$ (95\% CI 3.2-4.6\%) change in COPD mortality.

\section{Discussion}

The relative increased burden from COPD mortality compared with mortality from other causes is largely due to changes in population structure. The apparent paradox that COPD has risen to be the third most common cause of death at a time when age-standardised mortality rates from COPD have been falling is explained by the relatively faster decline in all-cause mortality. Between 1990 and 2010 the total number of COPD deaths fell from 3 to 2.8 million, a fall of 5.5\%, while during the same period deaths due to all causes fell by $17 \%$. The increase in COPD mortality due to population ageing explains why the absolute number of COPD deaths has decreased only slightly although age- and sex-specific mortality rates have been decreasing in almost all regions. Much of this decline is associated with changes in GNI, with countries with the greatest improvement in GNI having the greatest relative improvement in COPD mortality rates. Changes over time in the cumulative exposure to cigarette smoking have had relatively little impact. 
TABLE 2 Gross national income (GNI) per capita and smoking index by region and year

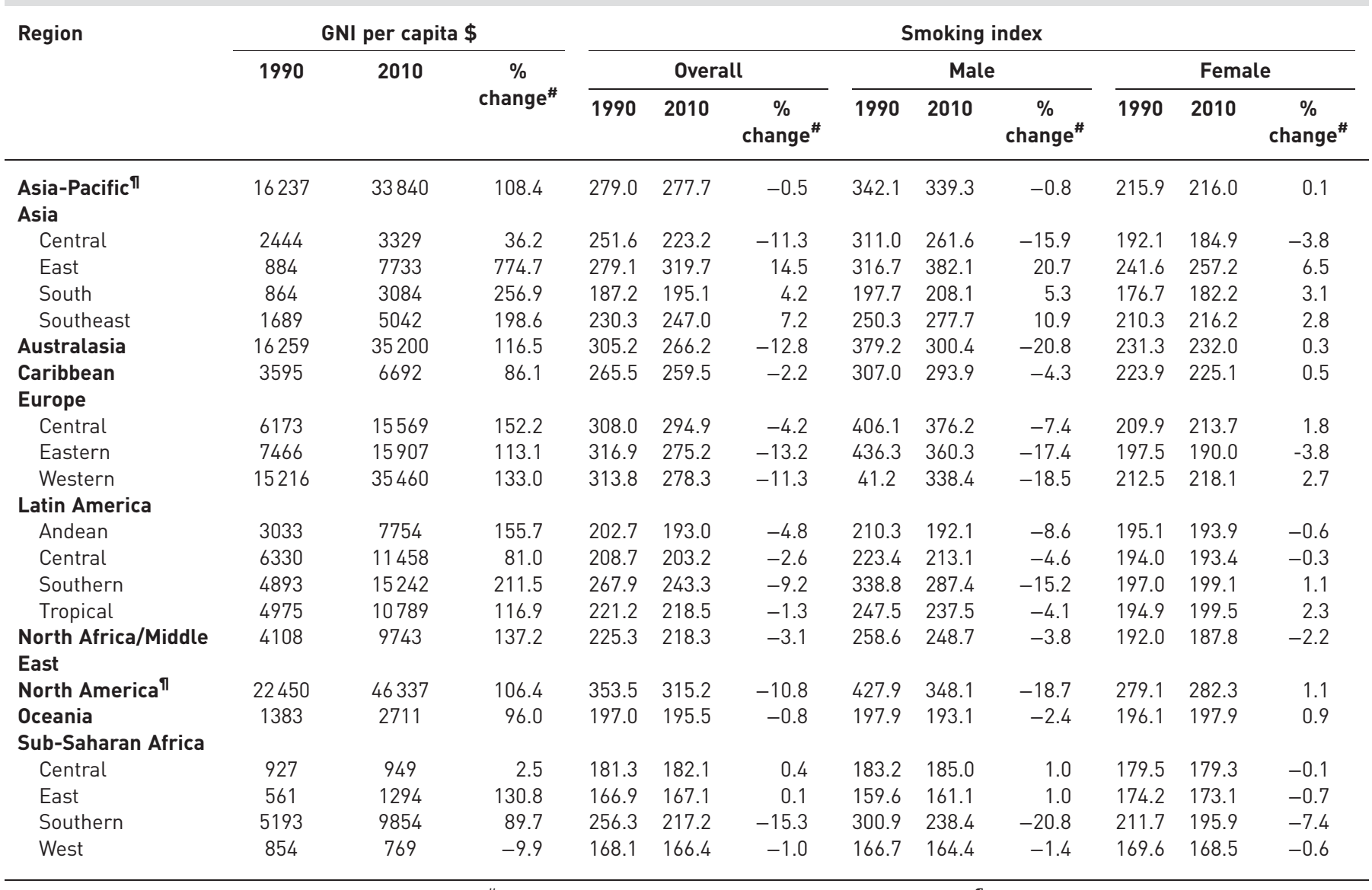

Data are presented as $n$, unless otherwise stated. ${ }^{*}$ : calculated as (value $2010-$ value $_{1990} /$ value $_{1990}$ ) $\times 100 ;{ }^{~}{ }^{\text {I: }}$ high income.

The ecological design of this study limits conclusions to inferences about populations rather than individuals but, unlike most ecological studies, the longitudinal design and analysis of within-region changes over time largely overcomes the usual problem of confounding with other large between-region differences, which is typical of many ecological studies.

Since the middle of the 19th century, the analysis of cause of death data has provided important insights into disease burden and the causes of ill health [19]. The data are nevertheless limited by incomplete records and inaccuracies in disease coding. Our definition of COPD mortality is deliberately broad, including all chronic lower respiratory tract causes whether bronchitic or obstructive, with the sole exception of asthma. Narrower definitions are more prone to misclassification and to local variation in the practice of both medicine and cause of death coding. With chronic diseases in particular, the assumption of a single underlying cause of death is often unrealistic, and those certifying the cause of death generally have limited information on which to determine the underlying cause of death and limited options on what cause to code it to. Poor practice often exacerbates these limitations. The protocols of the Global Burden of Disease programme have been developed to minimise these disadvantages, and the algorithms used to improve the reliability of these data, taking account of changes in the quality of cause of death coding and changes in the International Classification of Disease over time [15], have been extensively tested by out-of-sample tests of their predictive power.

The smoking index used provides a summary of smoking experience during a lifetime for each age group and has been extensively used, with the original paper being widely cited [16]. However, the estimates of cigarette smoking are indirect and depend on a high and consistent proportion of lung cancer deaths being attributable to smoking. This is an approximation. The population attributable fraction of lung cancer in men associated with smoking has been estimated as $92 \%$ in industrialised regions but as only $67 \%$ in developing regions [20]. Although the method would be improved by using local estimates of "nonsmoking" lung cancer, these are not universally available thus we have kept to a single estimate. Any 
TABLE 3 Results of the multilevel regression models for chronic obstructive pulmonary disease (COPD) mortality rate and the COPD mortality rate ratio

$\log$ COPD mortality rate

\begin{tabular}{|c|c|c|c|c|c|c|}
\hline & \multirow{2}{*}{\multicolumn{2}{|c|}{1990}} & \multirow{2}{*}{\multicolumn{2}{|c|}{2010}} & \multicolumn{2}{|c|}{ for 2010:1990 } \\
\hline & & & & & \multirow[t]{2}{*}{ Coefficient $(95 \% \mathrm{CI})$} & \multirow[t]{2}{*}{ p-value } \\
\hline & Coefficient $(95 \% \mathrm{Cl})$ & p-value & Coefficient $(95 \% \mathrm{CI})$ & p-value & & \\
\hline $\log \mathrm{GNI}$ & $-0.47(-0.68--0.26)$ & $<0.001$ & $-0.31(-0.53--0.08)$ & 0.01 & & \\
\hline log smoking index ratio for 2010:1990 & & & & & $0.004(0.002-0.004)$ & $<0.001$ \\
\hline $\log$ GNI ratio for 2010:1990 & & & & & $-0.34(-0.60--0.06)$ & 0.02 \\
\hline $45-49$ & $0.69(0.56-0.81)$ & $<0.001$ & $0.72(0.58-0.86)$ & $<0.001$ & $0.04(-0.04-0.11)$ & 0.38 \\
\hline $50-54$ & $1.47(1.35-1.59)$ & $<0.001$ & $1.47(1.34-1.61)$ & $<0.001$ & $0.01(-0.07-0.09)$ & 0.76 \\
\hline $55-59$ & $2.16(2.03-2.28)$ & $<0.001$ & $2.19(2.05-2.32)$ & $<0.001$ & $0.05(-0.03-0.13)$ & 0.24 \\
\hline $60-64$ & $2.81(2.68-2.94)$ & $<0.001$ & $2.88(2.74-3.01)$ & $<0.001$ & $0.10(0.02-0.18)$ & 0.02 \\
\hline $65-69$ & $3.60(3.47-3.72)$ & $<0.001$ & $3.76(3.62-3.89)$ & $<0.001$ & $0.19(0.11-0.27)$ & $<0.001$ \\
\hline $70-74$ & $3.91(3.78-4.04)$ & $<0.001$ & $4.08(3.94-4.22)$ & $<0.001$ & $0.20(0.12-0.28)$ & $<0.001$ \\
\hline Female & $-0.56(-0.62--0.50)$ & $<0.001$ & $-0.64(-0.70--0.57)$ & $<0.001$ & $-0.12(-0.16--0.07)$ & $<0.001$ \\
\hline
\end{tabular}

GNI: gross national income; Ref.: reference. bias introduced by this simplification would have only small effects on within-region comparisons between 1990 and 2010. Using this index is better than attempting to reconstruct smoking exposure over a lifetime from alternative sources such as surveys and tobacco sales, providing a relatively consistent source of information on the cumulative lifetime exposure to tobacco.

The downward trend for age- and sex-specific mortality rates from COPD have been demonstrated for the UK. MARKS AND BURNEY [7] reviewed mortality rates for respiratory causes of death from the beginning of the 20th century and showed that mortality rates began to decline around the start of the First World War. The effect of the smoking epidemic was to slow, or halt, this decline rather than lead to an absolute increase in rates. AdAIr et al. [11] and ERBAS et al. [12] showed similar results in Australia, with much lower and declining rates in the first half of the 20th century, followed by increasing rates up to 1970-1990 among men and 1990-2000 among women. PRIDE and SORIANO [8] also noted falling rates for bronchitis between 1931 and 1965 among 45-64 year olds in the UK, at a time when death rates from lung cancer were increasing. The strong association between COPD mortality and poverty is not new. In the UK this association was demonstrated at an ecological level by BARKER et al. [5] and was shown to be far stronger than the association between poverty and lung cancer. At an individual level, the social class gradient of the mortality rate for COPD has been steeper than those for lung cancer, or even for tuberculosis [21].

The strong association we observed between changes in COPD mortality and GNI and the relatively weak association with changes in smoking index depend on the rate of change in GNI and smoking index, as well as on the strength of their association with COPD mortality. The positive association of COPD mortality with the smoking index in both 2010 and 1990, and the positive association between changes in the smoking index and changes in COPD mortality, confirm the relevance of cigarette smoking to COPD mortality rates. The relatively small impact of smoking changes is largely due to the small change in global smoking rates, with improvements in some regions being offset by major increases in smoking in the populous regions of South, East and South-East Asia. Nevertheless the far greater impact of GNI requires some further explanation.

The BOLD study has shown a strong ecological association between the prevalence of airway obstruction and the prevalence of smoking, but has shown no such association with poverty. However, it did show a strong association between the prevalence of poverty and the prevalence of a low FVC, and the prevalence of a low FVC increases sharply in countries with a per capita GNI $<\$ 15000$ per annum [6]. Data from the USA have also shown that survival is much more strongly linked to FVC than to forced expiratory volume in $1 \mathrm{~s} / \mathrm{FVC}$, with the latter showing very little association with mortality once smoking had been adjusted 
for [22]. Although some discount the low FVC in poor countries as being confounded by race, evidence from the USA shows that the outcome from a low FVC is no different in African-Americans and White Americans after adjusting for age, sex and height [23]. These studies suggest that the high mortality from COPD in low-income countries is associated with a low FVC and not obstruction, as has generally been assumed, and raises an important question over the origins of this low FVC. There is good evidence that low ventilatory function in later life is associated with low birthweight $[5,24-27]$ and possibly with early infections $[4,5,28]$, and these conditions are associated with poverty $[4,29,30]$. However, COPD mortality appears to respond immediately to improvements in GNI, and it is therefore unlikely that the changes noted here are solely related to effects from poverty in early life. The mechanism of this more immediate effect and whether it is mediated through general changes in lifestyle, including environmental changes, or through access to healthcare is unclear. It is unlikely that it is due to specific changes in care for COPD, but general access to support could be part of an explanation.

These findings have important implications for both policy and research. The association between COPD mortality and smoking emphasise the need to reduce smoking rates in all regions, particularly those with increasing smoking rates, but our results show that this will not be enough. The strong association with poverty, particularly in the poorest regions of the world, demonstrate that poverty reduction will be critical in reversing the growing importance of COPD mortality. In the meantime, further research is required to understand what mediates the effects of poverty on COPD mortality.

In conclusion, the increase in the global burden of COPD mortality between 1990 and 2010 has been due to changes in the age distribution, while improved economic conditions, particularly in the poorest countries, have been associated with a fall in age- and sex-specific COPD mortality rates. The change in global smoking rates has been relatively small over the past 20 years, with decreases in some areas offset by increases in others. This accounts for the relatively small influence of changes in smoking habits on global COPD mortality changes.

\section{Acknowledgements}

We would like to thank Christopher Murray, Rafael Lozano, Nicholas J. Kassebaum and Spencer L. James (all Institute for Health Metrics and Evaluation, Seattle, WA, USA) for their contributions.

\section{References}

1 Lozano R, Naghavi M, Foreman K, et al. Global and regional mortality from 235 causes of death for 20 age groups in 1990 and 2010: a systematic analysis for the Global Burden of Disease Study 2010. Lancet 2012; 380: 2095-2128.

2 Murray CJ, Lopez AD. Alternative projections of mortality and disability by cause 1990-2020: Global Burden of Disease Study. Lancet 1997; 349: 1498-1504.

3 Eisner M, Anthonisen N, Coultas D, et al. An official American Thoracic Society public policy statement: novel risk factors and the global burden of chronic obstructive pulmonary disease. Am J Respir Crit Care Med 2010; 182: 693-718.

4 Barker DJ, Osmond C. Childhood respiratory infection and adult chronic bronchitis in England and Wales. BMJ 1986; 293: 1271-1275.

5 Barker DJ, Godfrey KM, Fall C, et al. Relation of birth weight and childhood respiratory infection to adult lung function and death from chronic obstructive airways disease. BMJ 1991; 303: 671-675.

6 Burney P, Jithoo A, Kato B, et al. Chronic obstructive pulmonary disease mortality and prevalence: the associations with smoking and poverty - a BOLD analysis. Thorax 2014; 69: 465-473.

7 Marks G, Burney P. Diseases of the respiratory system. In: Charlton J, Murphy JM, ed. The Health of Adult Britain: 1841-1994. Volume 2. London, The Stationery Office, 1997; pp. 93-113.

8 Pride NB, Soriano JB. Chronic obstructive pulmonary disease in the United Kingdom: trends in mortality, morbidity and smoking. Curr Opin Pulm Med 2002; 8: 95-101.

9 Lopez AD, Shibuya K, Rao C, et al. Chronic obstructive pulmonary disease: current burden and future projections. Eur Respir J 2006; 27: 397-412.

10 Tan WC, Seale P, Ip M, et al. Trends in COPD mortality and hospitalizations in countries and regions of Asia-Pacific. Respirology 2009; 14: 90-97.

11 Adair T, Hoy D, Dettrick Z, et al. 100 years of mortality due to chronic obstructive pulmonary disease in Australia: the role of tobacco consumption. Int J Tuber Lung Dis 2012; 16: 1699-1705.

12 Erbas B, Ullah S, Hyndman RJ, et al. Forecasts of COPD mortality in Australia: 2006-2025. BMC Med Res Method 2012; 12, 17.

13 Pham T, Ozasa K, Kubo T, et al. Age-period-cohort analysis of chronic obstructive pulmonary disease mortality in Japan, 1950-2004. J Epidemiol 2012; 22: 302-307.

14 López-Campos JL, Ruiz-Ramos M, Soriano JB. Mortality trends in chronic obstructive pulmonary disease in Europe, 1994-2010: a joinpoint regression analysis. Lancet Respir Med 2014; 2: 54-62.

15 Naghavi M, Makela S, Foreman F, et al. Algorithms for enhancing public health utility of national causes-of-death data. Popul Health Metr 2010; 8: 9.

16 Peto R, Lopez AD, Boreham J, et al. Mortality from tobacco in developed countries: indirect estimation from national vital statistics. Lancet 1992; 339: 1268-1278.

17 The World Bank. GNI per capita, PPP (current international \$). http://data.worldbank.org/indicator/NY.GNP. PCAP.PP.CD. Date last accessed: June 6, 2012. 
18 Williams R. Using the margins command to estimate and interpret adjusted predictions and marginal effects. Stata J 2012; 12: 308-331.

19 Greenhow EH. Papers relating to the sanitary state of the people of England: being the results of an inquiry into the different proportions of death produced by certain diseases in different districts of England. London, H.M. Stationery Office, 1858.

20 Ezzati M, Lopez AD. Estimates of global mortality attributable to smoking in 2000. Lancet 2003; 362: 847-852.

21 Population Censuses and Surveys Office. Occupational mortality: the registrar general's decennial supplement for Great Britain, 1979-80, 1982-83. London, H.M. Stationery Office, 1986.

22 Burney PG, Hooper R. Forced vital capacity, airway obstruction and survival in a general population sample from the USA. Thorax 2011; 66: 49-54.

23 Burney PG, Hooper RJ. The use of ethnically specific norms for ventilatory function in African-American and white populations. Int J Epidemiol 2012; 41: 782-790.

24 Lawlor DA, Ebrahim S, Davey Smith G. Association of birth weight with adult lung function: findings from the British Women's Heart and Health Study and a meta-analysis. Thorax 2005; 60: 851-858.

25 Qi-Qiang H, Tze-Wai W, Lin D, et al. Birth weight and lung function in a cohort of Chinese school children. Pediatr Pulmonol 2009; 44: 662-668.

26 Canoy D, Pekkanen J, Elliott P, et al. Early growth and adult respiratory function in men and women followed from the fetal period to adulthood. Thorax 2007; 62: 396-402.

27 Pei L, Chen G, Mi J, et al. Low birth weight and lung function in adulthood: retrospective cohort study in China, 1948-1996. Pediatrics 2010; 125: e899-e905.

28 Shaheen SO, Barker DJ, Shiell AW, et al. The relationship between pneumonia in early childhood and impaired lung function in late adult life. Am J Respir Crit Care Med 1994; 149: 616-619.

29 Kramer MS, Seguin L, Lydon J, et al. Socioeconomic disparities in pregnancy outcome: why do the poor fare so poorly? Paediatr Perinat Epidemiol 2000; 14: 194-210.

30 Thorn LK, Minamisava R, Nouer SS, et al. Pneumonia and poverty: a prospective population-based study among children in Brazil. BMC Infect Dis 2011; 11: 180. 